\title{
Expression of Cyclooxygenase-1 and Cyclooxygenase-2 in normal and pathological human oral mucosa
}

\author{
Annamaria Mauro $^{1 *}$, Luana Lipari ${ }^{1 *}$, Angelo Leone ${ }^{1}$, Silvia Tortorici ${ }^{2}$, \\ Francesco Burruano ${ }^{2}$, Salvatore Provenzano ${ }^{1}$, Aldo Gerbino ${ }^{1}$ and Maria Buscemi ${ }^{1}$ \\ ${ }^{1}$ BioNec, Dipartimento di Biomedicina Sperimentale e Neuroscienze Cliniche, Facoltŕ di Medicina \\ e Chirurgia, Universitŕ di Palermo, Palermo, Italy \\ ${ }^{2}$ Dipartimento di Chirurgia Orale e Maxillo Facciale, Facoltŕ di Medicina e Chirurgia, \\ Universitŕ di Palermo, Palermo, Italy
}

\begin{abstract}
Cyclooxigenase (COX) is the rate-limiting enzyme for the conversion of arachidonic acid (AA) to prostaglandins (PGs). Two isoforms of COX have been identified: COX-1 is constitutively expressed in many cells and is involved in cell homeostasis, angiogenesis and cell-cell signalling; COX-2 is not expressed in normal condition however it is strongly expressed in inflammation. The oral cavity is costantly exposed to physical and chemical trauma that could lead to mucosal reactions such as hyperplasia, dysplasia and cancer. Early diagnosis is the most important issue to address for a positive outcome of oral cancer; therefore it would be useful to identify molecular markers whose expression is associated with the various stages of oral cancer progression. Since COX enzyme has been involved, with different mechanisms, in the development and progression of malignancies we decided to investigate the expression and localization of COX-1 and COX-2 in normal human oral mucosa and three different pathologies (hyperplasia, dysplasia and carcinoma) by immunohistochemistry and RT-PCR. COX-1 mRNA and protein have been detected already in normal oral mucosa and their expression progressively increases from normal samples towards hyperplasia, dysplasia and finally carcinoma. On the contrary, COX-2 is not expressed in the normal tissue, starts to be expressed in hyperplasia, reaches the maximum activation in dysplasia and then starts to be downregulated in carcinoma.
\end{abstract}

Key words: cyclooxigenases, human oral mucosa, hyperplasia, dysplasia, carcinoma.

\section{Introduction}

Cyclooxigenase (COX), also known as prostaglandinendoperoxide synthase (PTGS), is the rate-limiting enzyme that catalyses the first step in prostaglandin (PG) biosynthesis and acts both as a dioxygenase and as a peroxidase. It catalyses the conversion of arachidonic acid (AA) to the key upstream prostanoid precursor prostaglandin $\mathrm{H}_{2}\left(\mathrm{PGH}_{2}\right)$ which is metabolised into the prostaglandin isoforms $\mathrm{PGE}_{2}, \mathrm{PGD}_{2}, \mathrm{PGF}_{2}$, $\mathrm{PGI}_{2}$, or thromboxane $\mathrm{A}_{2}\left(\mathrm{TXA}_{2}\right)$.

Two isoforms of $\mathrm{COX}$ have been identified: the constitutive COX-1 and the inducible COX-2, which

Correspondence: Prof. Aldo Gerbino, BioNec, Dipartimento di Biomedicina Sperimentale e Neuroscienze Cliniche,

Facoltŕ di Medicina e Chirurgia, University of Palermo

Via del Vespro 129, 90127 Palermo (I).

Tel. 0039-091-6553584, Fax. 0039-091-6553586

e-mail: aldogerbino@tiscali.it differ in the regulation of their expression and tissue distribution [1].

COX-1 is constitutively expressed in a broad range of cells and tissues where it mediates the synthesis of PGs required for physiological functions. COX-1 regulates angiogenesis, it is also thought to be involved in cell-cell signaling and in the maintenance of tissue homeostasis.

COX-2 is normally undetectable in most tissues, although there are some exceptions, for example seminal vesicles, kidney and regions of the central nervous system (hippocampus, hypothalamus and spinal cord) contain constitutively high levels of COX-2. COX-2 is rapidly induced by various mitogenic stimuli and proinflammatory agents including cytokines and endotoxin [1-3]. COX-2 is expressed in a limited number of cell types and is regulated by specific stimulatory events,

\footnotetext{
${ }^{*}$ These two authors contributed equally to the work.
} 
suggesting that it is responsible for the prostanoid biosynthesis required for inflammation and mitogenesis.

Recently, over-expression of COX-2 was observed in a variety of human malignancies, including colonic, gastric, breast, esophageal, bronchial, prostatic carcinomas, and oral cancer [4-10]. Many studies indicated that COX-2 contributes to tumorigenesis and to the development of malignant phenotype through several mechanisms, such as (1) inhibition of apoptosis; (2) increased angiogenesis; (3) increased invasiveness; (4) modulation of inflammation/immuno-suppression; and (5) stimulation of cell proliferation [11-13].

Inflammation is a non-specific immune response to infection, irritation or other injury, it is characterized by redness, swelling, pain, and loss of function. When harmful agents invade the human body, inflammatory mediators are released by immune cells, this release causes vasodilatation, migration of neutrophils, chemotaxis and increased vascular permeability. Two separate pathways produce inflammatory mediators: one is initiated by COX-2 and produces $\mathrm{PG}_{\mathrm{s}}$, while the other is initiated by 5-Lypoxigenase (5-LOX) and produces $\mathrm{LT}_{\mathrm{s}}$, PGE2, LTA4 and LTB4 [14-17].

The oral cavity is exposed to chronic or recurrent, physical and chemical trauma that could lead to mucosal reactions such as hyperplasia, dysplasia and cancer. Early diagnosis has been indicated as the most important issue to address in the prevention of oral cancer, but the diagnosis of oral cancer at an early stage is made obstructed by the difficulty in differenciating premalignant and malignant lesions from similar-looking benign lesions. Early-stage oral cancers are asymptomatic, furthermore, they often appear innocuous, since the classic clinical features associated with advanced oral cancers, including ulceration, induration, elevation, bleeding and cervical adenopathy usually are absent in early stage lesions.

Early diagnosis of precancerous oral lesions can have an important impact on oral cancer mortality rates. To this aim it could be useful to identify molecular markers whose expression is associated with the various stages of oral cancer progression.

In previous works we studied the expression of remodelling enzymes, such as metalloproteinases (MMPs) 2 and 9, in normal and pathological oral mucosa investigating the relationship between the level of expression of MMPs and the progression of oral mucosa pathological conditions towards the malignant phenotype [18-19]. Since COX enzyme has been involved, with various mechanisms, in the development of malignancies, in this work we investigated the expression and localization of COX-1 and COX-2 in normal human oral mucosa and three different pathological conditions (hyperplasia, dysplasia and carcinoma) by immunohistochemistry and RT-PCR.

\section{Materials and methods}

Samples. The samples used in this study were provided by the odontostomatological clinic of the University hospital "Paolo Giaccone" in Palermo and include: 9 normal oral mucosa, 13 cases of hyperplasia, 8 cases of dysplasia and 7 cases of carcinoma (4 cases of squamous cell carcinoma of the oral mucosa and 3 cases of squamous cell carcinoma of the tongue).

With the term hyperplasia we mean a pathological condition characterized by a hyperproliferation of normal cells, while for dysplasia we refer to a pathology characterized by disorganized hyperproliferation of cells that present some grade of atypia and leads to altered tissue morphology, it is a pre-cancerous lesion that can regress or evolve toward a cancer. In the carcinoma group we include malignant tumors of epithelial origin.

Immunohistochemistry. Samples were dissected and fixed in formalin solution. After fixation the tissue was dehydrated in a series of alcohols, cleared in xylene and paraffin embedded. Section of $7 \mu \mathrm{m}$ were cut with a Leica microtome RM2145, dried overnight at $37^{\circ} \mathrm{C}$ and then stored at room temperature until use. On the day of the experiment slides were dewaxed in xylene and rehydrated in a series of alcohols. Slides were then transferred into distilled water for $5 \mathrm{~min}$.

The immunohistochemistry was performed using the "DakoCytomation EnVision + System-HRP (AEC)" kit from Dako (Dako, Glostrup Denmark), following the manufacturer's instructions. Briefly: sections were incubated with the "Peroxidase block" reagent for $5 \mathrm{~min}$ at room temperature. The samples were rinsed once in PBS (Phosphate Buffer Saline) pH 7.2. The sections were covered with antibody solution and incubated at $4^{\circ} \mathrm{C}$ overnight.

Monoclonal mouse anti human COX-1 antibody (ZyMed Laboratories, Invitrogen immunodetection) (1:50 dilution), monoclonal mouse anti human COX-2 (DakoCytomation) (1:100 dilution) were used. As negative controls for our antibodies we used mouse IgG1 antibody (Dako, clone DAK-G01) (1:100 dilution). All antibodies were diluted in a $0.1 \%$ Bovine Serum Albumine (BSA) solution.

Human seminal vesicles and human epididymis were used respectively as positive control samples for COX-2 and COX-1 antibodies since these proteins are constitutively expressed in these tissues.

Samples were rinsed twice in PBS pH 7.2 and then incubated for 30 min. with the "Peroxidase Labelled Polymer" reagent. Samples were rinsed twice in PBS pH 7.2. They were then incubated for $4 \mathrm{~min}$. with the "Substrate-Chromogen" reagent. The staining procedure was identical for all the samples. The same incubation time with the chromogen reagent was used for all the samples. Reaction was stopped by rinsing the slides in distilled water.

A negative control sample was treated in an identical manner but the primary antibody was omitted. Slides were coverslipped using the "DakoCytomation Faramount Aqueous Mounting Medium" from Dako (Dako, Glostrup Denmark). The specimens were observed under a Leica DM1000 light microscope.

Semi-quantitative evaluation of immunoreactivity. The intensity of immunostaining for COX-1 and COX-2 in our specimens was evaluated by three independent observers according to the following criteria: (0) no staining, (1) light staining, (2) moderate staining, (3) intense staining. Statistical analysis of data was performed by the chisquare test, and P-value less than 0.05 was taken to be significant.

Total RNA extraction. Samples were frozen in liquid nitrogen immediately after surgical dissection and stored at $-80^{\circ} \mathrm{C}$ until use. Total RNA extraction was accomplished using the "illustra RNAspin Mini Kit" (Amersham Biosciences, Milan, Italy) following the manufacturer's instructions. RNA yield was evaluated spectrophotometrically (A260/A280) and RNA aliquots were stored at $-80^{\circ} \mathrm{C}$ until use. 
Table 1. Name, sequence and amplification product size of the primers used in this study.

\begin{tabular}{|c|c|c|}
\hline Primer name & Primer sequence & Product size \\
\hline COX-1 human forward & $\triangle \wedge \mathrm{G} T \wedge \mathrm{C} \mathrm{C} \wedge \mathrm{G} G \mathrm{GTG} \mathrm{CTG} G \wedge \mathrm{T} \mathrm{GG}$ & 319 base pairs \\
\hline COX-1 human reverse & $\mathrm{GCT} G \mathrm{GC} \wedge \mathrm{GG} \wedge \wedge \wedge \mathrm{T} \wedge \mathrm{GC} \mathrm{C} \wedge \mathrm{C} \mathrm{TC}$ & \\
\hline COX-2 human forward & CCA CCC GCA GIA CAG AAA GI & 196 base pairs \\
\hline COX-2 human reverse & CAG GAl ACA GCI CCA CAG CA & \\
\hline GAPDII human forward & GAG TCA ACG GAT T"IG GL GI & 238 basc pairs \\
\hline GAPLII human reverse & 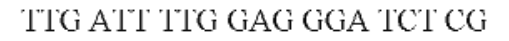 & \\
\hline
\end{tabular}

Reverse-Transcription (RT) - Polymerase Chain Reaction (PCR). For RT reaction $2 \mu \mathrm{g}$ RNA was used. To avoid DNA contamination in the RNA samples DNAse digestion was performed using "AMPD1 kit" (SIGMA). RT reaction was performed using the "Enhanced avian HS RT-PCR kit" (SIGMA) following the manufacturer's instructions. Briefly: $1 \mu 1$ random nonamers and $1 \mu 1$ anchored oligo (dT) 23 were added to the DNAse digestion product and incubated at $70^{\circ} \mathrm{C}$ for $10 \mathrm{~min}$ to denature the sample. Then $2 \mu 110 \times$ Buffer, $1 \mu 1$ deoxynucleotide mix, $1 \mu 1$ RNAse inhibitor, $1 \mu 1$ Enhanced AMV Reverse Transcriptase enzyme and DEPC water were added to the sample. The RT reaction was performed in $20 \mu \mathrm{l}$ total volume at $42^{\circ} \mathrm{C}$ for $50 \mathrm{~min}$, followed by $95^{\circ} \mathrm{C}$ for $5 \mathrm{~min}$ to inactivate the enzyme. The PCR was performed using the "PCR enzyme Selection Kit - High specificity" (Invitrogen) following the manufacturer's instructions. Briefly: $2 \mu \mathrm{l}$ of template DNA, $0.5 \mu \mathrm{l}$ of the Primers mix (200 nM final concentration) and $22.5 \mu \mathrm{l}$ of the "Platinum Super Mix" were mixed together. The reaction was initially cycled at $94^{\circ} \mathrm{C}$ for $3 \mathrm{~min}$. It then underwent 40 cycles at $94^{\circ} \mathrm{C}$ for $60 \mathrm{sec}$, at $56^{\circ} \mathrm{C}$ for $60 \mathrm{sec}$, at $72^{\circ} \mathrm{C}$ for $60 \mathrm{sec}$, with a final extension at $72^{\circ} \mathrm{C}$ for 10 minutes. GAPDH (GlycerAldeyde 3-Phosphate DeHydrogenase) gene was used as internal positive control. Primers sequences are listed in table 1 . PCR products were visualized on $2 \%$ agarose gels, stained with SYBR GREEN, observed with a transilluminator and photographed with a digital camera.

Ethical issues. Informed consent was obtained from the patients a pre-surgery. The experimentation was approved by the ethics committee.

\section{Results}

Immunohistochemical analysis of normal oral mucosa showed a very low immunoexpression for COX-1 in the epithelial region (Fig. 1A), while it did not show immunoreactivity for COX-2 (Fig. 2A). RT-PCR analysis performed on normal oral mucosa confirmed this data showing COX-1 mRNA transcription but not COX-2 (Fig. 3).

All hyperplasia samples showed COX-1 expression in mucosal epithelium (Fig. 1B) and vessel endothelium (Fig. 1C) and COX-1 immunoreactivity was significantly higher compared to control $(p<0.05)$ (Chart 1). Some of hyperplasia samples showed a weak COX2 expression in epithelium and vessel endothelium (Figs. 2B, 2C) showing the same spatial distribution as COX-1. Immunohistochemical data were confirmed by RT-PCR analysis; COX-1 mRNA was detected in all hyperplasia samples, while only part of the samples expressed COX-2 mRNA.
All dysplasia samples expressed both COX-1 (Figs. 1D and 1E) and COX-2 proteins (Figs. 2D, 2E). For both protein expression was localised in the cytoplasm of the cells and a clear zone was evident in the nuclear region. COX-1 immunoreactivity was more intense than hyperplasia samples and was localized in epithelium and around vessel walls. COX-2 expression was significantly higher than hyperplasia $(p<0.05)$. RTPCR analysis confirmed these data showing mRNA transcription for both genes; in figure 4 is evident an intense expression of both COX-1 and COX-2 even compared to the control gene GAPDH.

All carcinoma samples showed COX-1 expression, immunoreactivity was stronger than all the other normal and pathological samples examined (Fig.1F) and this increase was statistically significant $(p<0.05)$. COX-2 protein was expressed only in some of carcinoma and immunostaining was not intense as in dysplasia (Fig. 2F). RT-PCR analysis was in agreement with immunohistochemical data, showing an intense COX-1 mRNA expression in all carcinomas and a moderate COX-2 mRNA presence in some of the samples.

Immunohistochemistry showed a progressive increase of COX-1 protein expression from normal oral mucosa towards hyperplasia, dysplasia and finally carcinoma (Chart 1). On the contrary, semi-quantitative RT-PCR analysis showed a quite constant level of expression for COX-1 mRNA in normal samples, hyperplasia and dysplasia and an increase of COX-1 gene expression only in carcinoma.

Both immunohistochemistry and RT-PCR analysis gave the same results for COX-2 trend: COX-2 expression was not detected in normal oral mucosa samples, while a moderate expression was found in hyperplasia. The maximum COX-2 mRNA and protein levels were detected in dysplasia samples, while carcinoma shows a slight reduction of COX-2 expression (Chart 1).

\section{Discussion}

COX is the key-enzyme catalysing the first step in the biosynthesis of prostaglandins, molecules that play a pivotal role in the initiation and progression of many cancers. In particular COX-2 seems to act synergistically with cytosolic phospholipase A2, the 

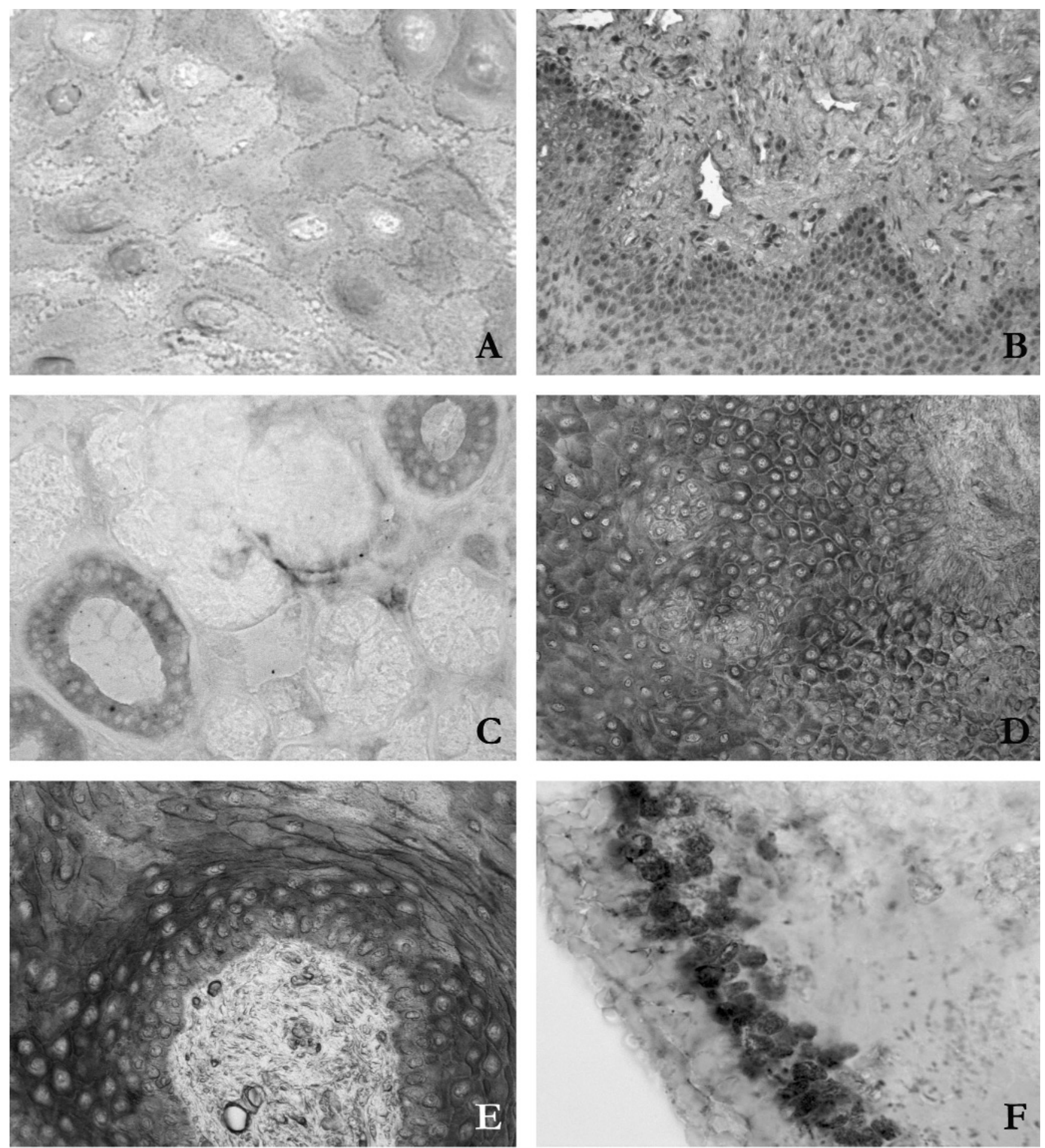

Fig. 1. COX-1 immunohistochemistry in human oral mucosa samples. (A) Light immunostaining for COX-1 in the epithelium of normal oral mucosa (original magnification $\times 100$ ); (B) Hyperplasia: COX-1 expression in epithelium and many cells of connective tissue (original magnification $\times 20$ ); (C) Hyperplasia: COX-1 in vessel endothelium (original magnification $\times 40$ ); (D-E) Dysplasia: COX-1 expression in the cytoplasm of epithelial cells (original magnification $\times 20, \times 40)$; (F) Carcinoma: very strong COX-1 expression in some cells of the superficial epithelial layers (original magnification $\times 100$ ).

other key enzyme of prostaglandin synthesis, to contribute to the process of oral carcinogenesis and both enzymes are overexpressed in oral dysplasia and carcinoma [8].
Overexpression of COX-2 is reported in many cancers and selective COX-2 inhibitors are known to suppress cell proliferation by inducing G0/G1 phase, cell cycle arrest and apoptosis [13,20-21]. 

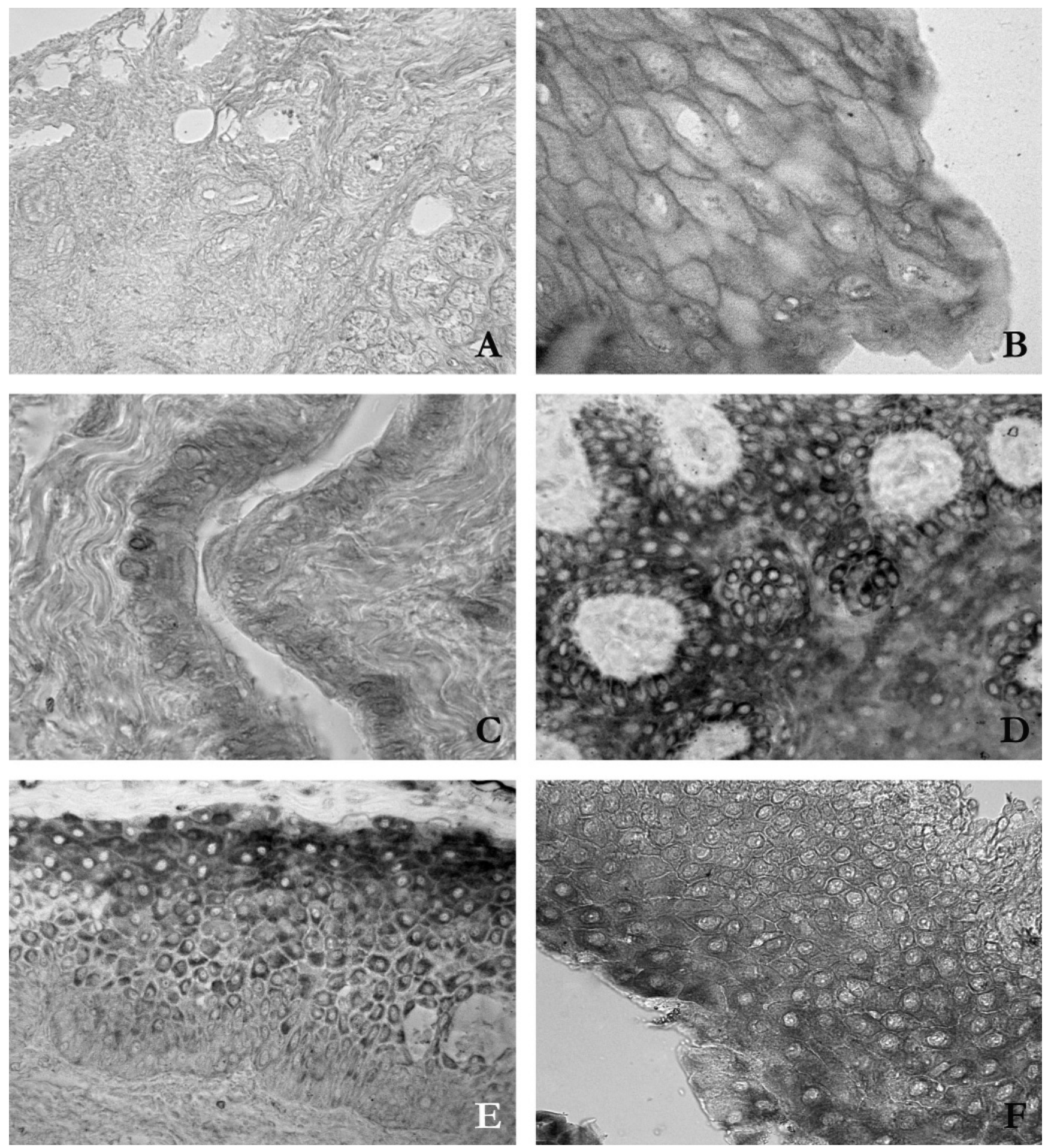

Fig. 2. COX-2 immunohistochemistry in human oral mucosa samples. (A) Normal oral mucosa: no COX-2 immunostaining; (B) Hyperplasia: light COX-2 expression in epithelium (original magnification $\times 40$ ); (C) Hyperplasia: light COX-2 expression in endothelium (original magnification $\times 100$ ); (D-E) Dysplasia: strong COX-2 immuno-expression in epithelium (original magnification $\times 40, \times 20$ ); (F) Carcinoma: COX-2 in the cytoplasm of epithelial cells (original magnification $\times 40$ ).

The contribution of COX to carcinogenesis is due to its involvement in several key-mechanisms: 1) the conversion of pro-carcinogens to carcinogens as a consequence of arachidonic acid metabolism, 2) stimulation of cell growth, 3 ) inhibition of apoptosis through P53 suppression and Bcl2 induction, 4) stimulation of VEGF and angiogenesis, 5) promotion of invasion and metastasis via matrix metalloproteinases 


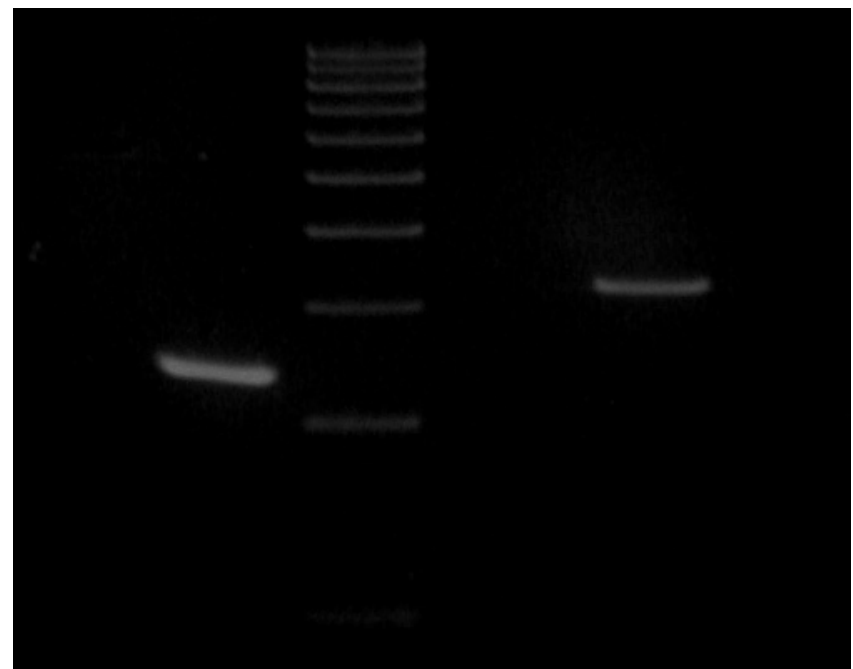

Fig. 3. Representative picture of RT-PCR analysis in normal oral mucosa sample. Lane 1: GAPDH amplification product (232 bp); lane 2: $100 \mathrm{bp}$ DNA ladder; lane 3: no amplification product for COX-2 gene; lane 4: COX-1 amplification product (319 bp).

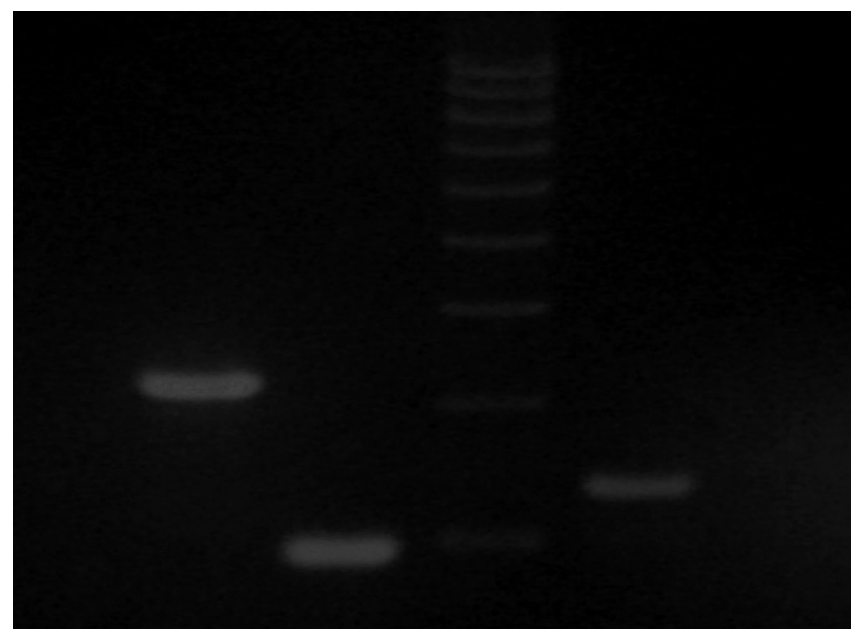

Fig. 4. Representative picture of RT-PCR analysis in dysplasia sample. Lane 1: COX-1 amplification product (319 bp); lane 2: COX-2 amplification product (196 bp); lane 3: 100bp DNA ladder; lane 4: GAPDH amplification product (232 bp).

induction, 6) immunosuppression by IL-10 induction [12-13,22-23].

As it is well known, COX-2 is probably one of the most important agents involved in the development and evolution of inflammation. Long term use of drugs that inhibit COX activity, such as aspirin or nonsteroidal anti-inflammatory drugs (NSAID), is associated with $30-50 \%$ reduction in risk of colorectal, esophagus, stomach, breast, lung, prostate, urinary bladder and ovary cancer. The fact that chronic or acute inflammation is commonly associated with cancer complicates the interpretation of COX-2 expression in cancer. On one hand, the tissue disruption and cell death typical of cancer recruit pro-inflammatory cells and lead to inflammation. On the other hand,

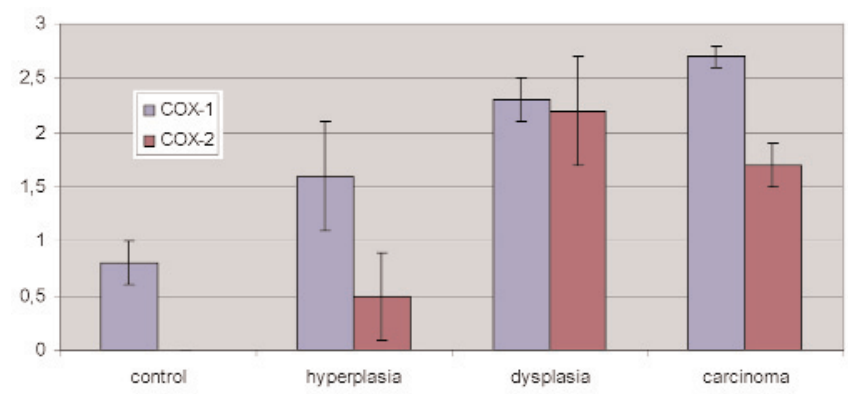

Fig. 5. Semi-quantitative analysis of COX-1 and COX-2 immunoreactivity in the samples. Bars represents the mean standard deviation.

some types of chronic inflammation are the cause of the initiation of certain cancers, such as chronic hepatitis, chronic gastritis and chronic ulcerative colitis. Thus it may be very difficult to separate COX-2 expression related to inflammation from that related to tumor transformation [24].

Inflammation is the main etyological factor in oral squamous cell carcinoma (OSCC), COX-2 activity is related both to inflammation and carcinogenesis and its expression has been found increased in OSCC together with other pro-inflammatory molecules as inducible nitric oxide synthase (iNOS) and vascular endothelial growth factor (VEGF); overexpression of these markers was also associated with limph node metastasis, tumor staging and grading [25-26].

Cohesive scientific evidence from molecular, animal, and human investigations support the hypothesis that aberrant induction of COX-2 and up-regulation of the prostaglandin cascade play a significant role in carcinogenesis. Blockade of these processes has strong potential for cancer prevention and therapy $[12,22,26]$.

The hypothesis of COX involvement in cancer progression has been strengthened by the effect of COX inhibitors that have been successfully used in the treatment of many cancers [27-31]. The efficacy of COX selective and nonselective inhibitors against oral carcinogenesis has been confirmed by in vitro and in vivo studies on rat: COX inhibitors showed to be chemopreventive agent decreasing oral cancer incidence, cell growth, proliferation of xenografted tumors, cancer invasion score and cancer-related mortality $[10,32]$. The mechanism responsible for these effects is still unclear, but some of the studies indicated a possible involvement of matrix metalloproteinases (MMPs) in this mechanism: COX inhibition suppresses the invasiveness of oral squamous cell carcinoma via down-regulation of MMP-2 activity [33-34]. Even if COX inhibitors are promising candidates for cancer therapy it is to consider the emerging side-effects of these drugs, above all the cardiovascular risk associated with the use of these compounds $[12,27]$. 
The use of COX inhibitors in treatment of cancer has given good results especially in combination with other conventional cancer therapy. The combination of COX inhibitors with chemotherapy or radiotherapy has shown synergistic effects on tumor growth and development both in vitro and in vivo [35-36]. Also the combination with EGFR inhibitor resulted in almost complete inhibition of tumor growth [37-38].

While the overexpression of COX-2 in oral cancer has been widely reported and is generally accepted by the scientific community, there are contrasting opinions on the possibility that COX-2 expression could be used as a prognostic tool to predict the outcome of the disease; some author report a correlation between COX-2 overexpression and poor prognosis [39] while others don't [40]. Moreover, opinions regarding the correlation between COX-2 expression and the histologic grade of oral cancer are highly conflicting; many authors report a progressive increase of COX-2 expression from normal oral mucosa towards invasive carcinoma [39,41-43], others report an higher COX-2 expression in premalignant lesion than in malignancies $[4,6,44-45]$, while others report the lack of correlation between COX-2 level and tumor stage or grade $[7,46]$.

Another confounding factor is the wide variability of COX-2 expression observed in different individuals in the same pathological condition, this heterogeneity has been attributed to the difference in oral mucosa microenvironment, evolutive stage of the tumor, etiologic factors and gene mutations.

In this study we examined the following three pathological conditions: hyperplasia, dysplasia and carcinoma, as sequential steps in the process of cancer progression towards the malignant phenotype. This approach can help understanding the development of tumor transformation from normal oral mucosa to oral cancer.

By immunohistochemistry we detected a progressive increase of COX-1 protein expression from normal oral mucosa towards hyperplasia, dysplasia and finally carcinoma; this increase parallels the severity of the lesion. On the contrary, RT-PCR analysis showed a quite constant level of COX-1 mRNA in normal samples, hyperplasia and dysplasia and an increase of COX-1 gene expression only in carcinoma.

These data suggest that the progressive increase of COX-1 enzyme detected through immunohistochemistry is due more to regulation of protein synthesis (translation) than regulation of gene expression (transcription). This means that the level of COX-1 gene expression in control, hyperplasia and dysplasia remains constant while the rate of protein synthesis can vary in relation with the transition from a physiological to a pathological condition. Finally in the last step of the transition to carcinoma the increase of COX-1 gene expression parallels the increase of protein expression.
Both immunohistochemistry and RT-PCR analysis gave the same results for COX-2 trend; COX-2 expression was not detected in normal oral mucosa, while a moderate expression was found in hyperplasia. The maximum COX-2 mRNA and protein levels were detected in dysplasia samples, while carcinoma showed a light reduction of COX-2 expression compared to dysplasia.

Our data are in agreement with all the studies $[4,5,7,23-24]$ that report a higher expression of COX2 in precancerous lesions than in malignancies and that hypothesize a correlation between COX-2 activation and the early stages of carcinogenesis.

We believe that COX-2 activity could be involved in the initial phases of the process of tumor transformation. We hypothesize that COX-2 gene and protein expression, that is absent in normal cells, is required during the early stages of carcinogenesis. They are switched on during hyperplasia (with some of the samples moderately expressing COX-2) and are fully activated during dysplasia (with all the samples clearly expressing COX-2). Later on, in more advanced stages of tumor development such as carcinoma, when the tumor is well established, the involvement of COX-2 activity could be less critical and for this reason COX2 starts to be down-regulated (with only part of the samples still expressing COX-2).

In a previous study [19] we investigated the expression of the metalloproteinases MMP-2 and MMP-9 in normal oral mucosa and in the same three pathological conditions we refer in this work. We detected a progressive increase of MMPs mRNA and protein expression from normal oral mucosa to hyperplasia, dysplasia and carcinoma, finding a correlation between MMPs overexpression and the development of a malignant phenotype.

Starting from these data we moved our attention on COX-1 and COX-2 expression in tumor development. These two classes of enzymes (metalloproteinases and cycloxigenases) are both involved in phenomena related to inflammation, atherosclerosis, angiogenesis, cancer progression and invasiveness, and both are de-regulated in many types of tumor. Some studies indicated a possible involvement of MMPs in the control of cancer progression dependent by COX inhibitors [33-34]. So we thought it could be appropriate to consider these enzymes as if they were functionally correlated and study them in parallel.

Comparing the expression of MMPs and COXs in the samples examinated we found that COX-1 expression parallel that of MMP-2 and 9, increasing from normal oral mucosa towards carcinoma, while COX-2 does not follow the same trend, reaching its maximum expression in pre-cancerous conditions (dysplasia).

We hypothesize that extracellular matrix remodelling due to MMPs activity is a factor necessary for 
tumor establishment and progression, thus the increase of MMPs expression is maintained till the last steps of carcinogenesis. On the other hand, COX-2 pro-inflammatory activity, however, could be more involved in pre-cancerous conditions than in established tumor, so COX-2 over-expression can be more significant in dysplasia than in carcinoma. Finally the fact that COX-1 is inducible under inflammatory condition [21] could explain the progressive COX-1 increase we detected in the pathologies investigated.

This study, and further more, could contribute to the early diagnosis of oral cancer and to the development of new therapies based on cyclooxigenases inhibitors.

Aknowledgements: We thank Doctor Manjeet Shergill, dental surgeon from London for the kind language revision of this manuscript.

\section{References}

[1] Feng L, Sun W, Xia Y, et al. Cloning two isoforms of rat cyclooxygenase: differential regulation of their expression. Arch Biochem Biophys. 1993;307(2):361-8.

[ 2] Hla T and Neilson K. Human cyclooxygenase-2 cDNA. Proc Natl Acad Sci U S A. 1992;89(16):7384-8.

[3] Kujubu DA, Reddy ST, Fletcher BS and Herschman HR. Expression of the protein product of the prostaglandin synthase-2/ gene in mitogen-stimulated Swiss 3T3 cells. J Biol Chem. 1993;268:5425-30.

[4] Shamma A, Yamamoto H, Doki Y et al. Up-regulation of cyclooxygenase- 2 in squamous carcinogenesis of the esophagus. Clin Cancer Res. 2000;6(4):1229-38.

[5] Banerjee AG, Gopalakrishnan VK, Bhattacharya I and Vishwanatha JK. Deregulated cyclooxygenase-2 expression in oral premalignant tissues. Mol Cancer Ther. 2002;1(14): 1265-71.

[ 6] Shibata M, Kodani I, Osaki M et al. Cyclo-oxigenase-1 and2 expression in human oral mucosa, dysplasias and squamous cell carcinomas and their pathological significance. Oral Oncology. 2005;41:304-312.

[ 7] Pannone G, Sanguedolce F, De Maria S, Farina E, Lo Muzio $\mathrm{L}$, Serpico R, et al. Cyclooxygenase isozymes in oral squamous cell carcinoma:a real-time RT-PCR study with clinic pathological correlations. Int J Immunopathol Pharmacol. 2007;20(2):317-24.

[ 8] Zhang S, Du Y, Tao J, Wu Y, Chen N. Expression of cytosolic phospholipase A2 and cyclooxygenase 2 and their significance in human oral mucosae, dysplasias and squamous cell carcinomas. ORL J Otorhinolaryngol Relat Spec. 2008;70(4): 242-8.

[ 9] Sappayatosok K, Maneerat Y, Swasdison S, Viriyavejakul P, Dhanuthai K, Zwang J, et al. Expression of pro-inflammatory protein, iNOS, VEGF and COX-2 in oral squamous cell carcinoma (OSCC), relationship with angiogenesis and their clinico-pathological correlation. Med Oral Patol Oral Cir Bucal. 2009;14(7):E319-24.

[10] McCormick DL, Phillips JM, Horn TL, Johnson WD, Steele VE, Lubet RA. Overexpression of cyclooxygenase-2 in rat oral cancers and prevention of oral carcinogenesis in rats by selective and nonselective COX inhibitors. Cancer Prev Res (Phila Pa). 2010;3(1):73-81.

[11] Dempke W, Rie C, Grothey A and Schmoll HJ. Cyclooxygenase-2: a novel target for cancer chemotherapy? J Cancer Res Clin Oncol. 2001;127(7):411-7.
[12] Harris RE. Cyclooxygenase-2 (cox-2) and the inflammogenesis of cancer. Subcell Biochem. 2007;42:93-126.

[13] Ghosh N, Chaki R, Mandal V, Mandal SC. COX-2 as a target for cancer chemotherapy. Pharmacol Rep. 2010;62(2):233-44.

[14] Mizuno K, Yamamoto S and Lands WE. Effects of nonsteroidal anti-inflammatory drugs on fatty acid cyclooxygenase and prostaglandin hydroperoxidase activities. Prostaglandins. 1982;23:743-57.

[15] Terakado N, Shintani S, Yano J et al. Overexpression of cyclooxygenase-2 is associated with radioresistance in oral squamous cell carcinoma. Oral Oncology. 2004;40:383-389.

[16] Mizuno S, Kato K, Hashimoto A et al. Expression of cyclooxygenase-2 in Gastrointestinal Carcinoid Tumors. J Gastroenterol Hepatol. 2006;21(8):1313-1319.

[17] Klein T, Shephard P, Kleinert H and Kömhoff M. Regulation of cyclooxygenase-2 expression by cyclic AMP. Biochimica et Biophysica Acta. 2007;1773:1605-1618.

[18] Tortorici S, Mauro A, Burruano F et al. Matrix metalloproteinase-2 matrix metalloproteinase- 9 and inducible nitric oxide synthase in oral leukoplakia: immunohistochemistry and RT-PCR analysis. J Biol Regul Homeost Agents. 2008; 22(2):125-30.

[19] Lipari L, Mauro A, Tortorici S et al. Immunohistochemical and transcriptional expression of the matrix metalloproteinases MMP-2 and MMP-9 in normal and pathological human oral mucosa. J Biol Regul Homeostat Agents. 2009;23(4): 259-67.

[20] Choi EM, Heo JI, Oh JY et al. COX-2 regulates p53 activity and inhibits DNA damage-induced apoptosis. Biochem Biophys Res Commun. 2005;328(4):1107-12.

[21] Wang D and DuBois RN. Prostaglandins and cancer. Gut. 2006; $55: 115-122$

[22] De Souza Pereira R. Selective cyclooxygenase-2 (COX-2) inhibitors used for preventing or regressing cancer. Recent Pat Anticancer Drug Discov. 2009;4(2):157-63.

[23] Katoh H, Hosono K, Ito Y, Suzuki T, Ogawa Y, Kubo H, et al. COX-2 and prostaglandin EP3/EP4 signaling regulate the tumor stromal proangiogenic microenvironment via CXCL12-CXCR4 chemokine systems. Am J Pathol. 2010; 176(3):1469-83.

[24] Zha S, Yegnasubramanian V, Nelson WG, Isaacs WB and De Marzo AM. Cyclooxygenases in cancer: progress and perspective. Cancer Lett. 2004;215(1):1-20.

[25] Goulart Filho JA, Nonaka CF, da Costa Miguel MC, de Almeida Freitas R and Galvao HC. Immunoexpression of cyclooxygenase-2 and p53 in oral squamous cell carcinoma. Am J Otolaryngol. 2009;30(2):89-94.

[26] Singh P, Mittal A. Current status of COX-2 inhibitors. Mini Rev Med Chem. 2008;8(1):73-90.

[27] Fujimura T, Ohta T, Oyama K, Miyashita T, Miwa K. Cyclooxygenase-2 (COX-2) in carcinogenesis and selective COX-2 inhibitors for chemoprevention in gastrointestinal cancers. J Gastrointest Cancer. 2007;38(2-4):78-82.

[28] Breinig M, Schirmacher P, Kern MA. Cyclooxygenase-2 (COX-2)--a therapeutic target in liver cancer? Curr Pharm Des. 2007;13(32):3305-15.

[29] Shinohara N, Kumagai A, Kanagawa K, Maruyama S, Abe T, Sazawa A, et al. Multicenter phase II trial of combination therapy with meloxicam, a cox-2 inhibitor, and natural interferon-alpha for metastatic renal cell carcinoma. Jpn J Clin Oncol. 2009;39(11):720-6.

[30] Hu M, Peluffo G, Chen H, Gelman R, Schnitt S, Polyak K. Role of COX-2 in epithelial-stromal cell interactions and progression of ductal carcinoma in situ of the breast. Proc Natl Acad Sci USA. 2009;106(9):3372-7.

[31] Harris RE. Cyclooxygenase-2 (cox-2) blockade in the chemoprevention of cancers of the colon, breast, prostate, and lung. Inflammopharmacology. 2009;17(2):55-67. 
[32] Ohtsu N, Takaoka K, Segawa E, Hashitani S, Noguchi K, Kishimoto $\mathrm{H}$, et al. Antitumor effects of inhibitors of nitric oxide synthase or cyclooxygenase- 2 on human KB carcinoma cells overexpressing COX-2. Oncol Rep. 2010;24(1):31-6.

[33] Kwak YE, Jeon NK, Kim J, Lee EJ. The cyclooxygenase-2 selective inhibitor celecoxib suppresses proliferation and invasiveness in the human oral squamous carcinoma. Ann $N$ Y Acad Sci. 2007;1095:99-112.

[34] Kurihara Y, Hatori M, Ando Y, Ito D, Toyoshima T, Tanaka $\mathrm{M}$, et al. Inhibition of cyclooxygenase-2 suppresses the invasiveness of oral squamous cell carcinoma cell lines via downregulation of matrix metalloproteinase-2 production and activation. Clin Exp Metastasis. 2009;26(5):425-32.

[35] Pyo H, Choy H, Amorino GP, Kim JS, Cao Q, Hercules SK, et al. A selective cyclooxygenase-2 inhibitor, NS-398, enhances the effect of radiation in vitro and in vivo preferentially on the cells that express cyclooxygenase-2. Clin Cancer Res. 2001;7:2998-3005.

[36] Altorki NK, Keresztes RS, Port JL, Libby DM, Korst RJ, Flieder DB et al. Celecoxib, a selective cyclo-oxygenase-2 inhibitor, enhances the response to preoperative paclitaxel and carboplatin in early-stage nonsmall- cell lung cancer. $J$ Clin Oncol. 2003;21:2645-2650.

[37] Mann M, Sheng H, Shao J, Williams CS, Pisacane PI, Sliwkowski MX et al. Targeting cyclooxygenase 2 and HER$2 /$ neu pathways inhibits colorectal carcinoma growth. Gastroenterology. 2001;120:1713-1719.

[38] Kao J, Sikora AT, Fu S. Dual EGFR and COX-2 inhibition as a novel approach to targeting head and neck squamous cell carcinoma. Curr Cancer Drug Targets. 2009;9(8):931-7.

[39] Segawa E, Sakurai K, Kishimoto H, Takaoka K, Noguchi K, Hashitani S, et al. Expression of cyclooxygenase-2 and DNA topoisomerase II alpha in precancerous and cancerous lesions of the oral mucosa. Oral Oncol. 2008;44(7):664-71.
[40] Soland TM, Husvik C, Koppang HS, Boysen M, Sandvik L, Clausen OP, et al. A study of phosphorylated ERK1/2 and COX-2 in early stage (T1-T2) oral squamous cell carcinomas. J Oral Pathol Med. 2008;37(9):535-42.

[41] Nystrom ML, McCulloch D, Weinreb PH et al. Cyclooxygenase-2 inhibition suppresses alphavbeta6 integrin-dependent oral squamous carcinoma invasion. Cancer Res. 2006; 66(22):10833-42.

[42] Sakurai K, Urade M, Noguchi K et al. Prognostic significance of cyclooxygenase- 2 and DNA topoisomerase IIalpha expression in oral carcinoma. Head Neck. 2007;29(11): 1002-9.

[43] Sawhney M, Rohatgi N, Kaur J et al. Expression of NF-kappaB parallels COX-2 expression in oral precancer and cancer: association with smokeless tobacco. Int $J$ Cancer. 2007;120(12):2545-56

[44] Minter HA, Eveson JW, Huntley S, Elder DJ and Hague A. The cyclooxygenase 2-selective inhibitor NS398 inhibits proliferation of oral carcinoma cell lines by mechanisms dependent and independent of reduced prostaglandin E2 synthesis. Clin Cancer Res. 2003;9(5):1885-97.

[45] Saba NF, Choi M, Muller S et al. Role of cyclooxygenase-2 in tumor progression and survival of head and neck squamous cell carcinoma. Cancer Prev Res (Phila Pa). 2009; 2(9):823-9.

[46] Pandey M, Prakash O, Santhi WS, Soumithran CS and Pillai RM. Overexpression of COX-2 gene in oral cancer is independent of stage of disease and degree of differentiation. Int J Oral Maxillofac Surg. 2008;37:379-383.

Submitted: 24 May, 2010 Accepted after reviews: 11 August, 2010 